\title{
Reconstructed Target Range Profile via Unitary ESPRIT Superresolution Algorithm
}

\author{
Rui Zhang, ${ }^{1}$ Min Zhang, ${ }^{1}$ Xin Wang, ${ }^{2}$ and Zhaohui Cai ${ }^{1}$ \\ ${ }^{1}$ School of Physics and Optoelectronic Engineering, Xidian University, Xian 710071, China \\ ${ }^{2}$ Southwest China Research Institute of Electronic Equipment, Chengdu 610036, China \\ Correspondence should be addressed to Min Zhang; mzhang@mail.xidian.edu.cn
}

Received 28 April 2017; Accepted 17 July 2017; Published 15 August 2017

Academic Editor: Stefania Bonafoni

Copyright (C) 2017 Rui Zhang et al. This is an open access article distributed under the Creative Commons Attribution License, which permits unrestricted use, distribution, and reproduction in any medium, provided the original work is properly cited.

\begin{abstract}
We propose an efficient technique for target classification using one-dimensional high resolution range profile (HRRP). The proposed technique utilizes the unitary estimation of signal parameters via rotational invariance techniques (ESPRIT) algorithm to extract scattering centers and then reconstruct superresolution range profiles. Moreover, we employ the central moments to provide translation invariant and scale invariant feature vectors. Finally, the proposed unitary ESPRIT (U-ESPRIT) based range profile reconstruction method is applied to the simulated annealing resilient backpropagation (SARPROP) classification algorithm to evaluate the recognition performances. Recognition results using four different aircraft models are presented to assess the effectiveness of the proposed technique, and they are compared with those of the conventional range profiles obtained by fast Fourier transform (FFT). Comparison results on simulated data show that the HRRP reconstruction method is better than directly using HRRP in targets classification.
\end{abstract}

\section{Introduction}

High resolution range profile (HRRP) can reflect the radar radiation, that is, the scatters, which are projected onto the line of sight. Therefore, HRRP has wide applications in automatic target recognition (ATR) field since Li and Yang [1] proposed this for the first time.

However, there are two major challenges to use the range profile in target recognition. One is that it is highly aspect dependent and with large data storage. One solution to overcome this is to extract aspect invariant features from HRRP. Moments and functions of moments have been utilized as features in a number of applications to achieve invariant. There are several moments that could be used to guarantee the translation, scale, contrast, and rotation invariance including regular moments, Hu moments, Zernike moments, rotational moments, and complex moments. In this work, we propose only using the simple central moments to satisfy the translational invariance. The other challenge is resolution limitation, which significantly impacts the recognition results [2]. Several research works proposed improving the range resolution through superresolution techniques such as Multiple Signal Classification (MUSIC) [3], maximum likelihood [4], maximum entropy method [5], and Prony [6]. However, there are limited studies about their applications to target recognition. Reference [2] has used MUSIC in range profile recognition but suffers from several difficulties. First, the scattering number is set to a constant value of 30 , which may lead to scattering center mismatch. Second, the amplitude of MUSIC method cannot provide the amplitude of the range profile. Additionally MUSIC method has to involve an exhaustive searching procedure, thus requiring much more hardware for computation and storage.

Among several superresolution algorithms, unitary ESPRIT offers numerous advantages over other recently proposed ESPRIT [7] based closed-form techniques. First, the conjugation of observed data is used to extend the efficient data, which can increase the estimation accuracy of conventional least squares ESPRIT (LS-ESPRIT) or total least squares ESPRIT (TLS-ESPRIT) [8]. In addition, it is efficiently formulated in terms of real-valued computation throughout [9]. 
In this paper, unitary ESPRIT is successfully utilized in step frequency radar for scattering center extraction. Then we reconstruct the HRRP by means of the extracted scattering centers for target recognition. Moreover, due to the fact that there are only several isolated scattering centers at each aspect, the storage burden can be reduced in comparison with the conventional HRRP based classification method. At the same time, according to the reconstruction theory of the scattering centers, only the main components of signal are reconstructed; therefore better classification performance result can be achieved. Finally, classification performance under the same situation was compared between FFT based HRRP and reconstructed HRRP. The results of the range profiles of four targets show that unitary ESPRIT can get higher correct classification rate and is more robust to SNR.

\section{Principle of Target Recognition Algorithm}

2.1. Unitary ESPRIT for Scattering Center Extraction. The extraction of radar target scattering centers plays an important role in target classification, identification, recognition, and remote sensing fields. In this section, we briefly review the procedure of scattering center extraction method using unitary ESPRIT.

(1) Assuming a complex-valued data matrix $v_{N}(\psi)$ satisfies the invariance relationship given by

$$
e^{j \psi} J_{s 1} v_{N}(\psi)=J_{s 2} v_{N}(\psi)
$$

$J_{s 1}$ and $J_{s 2}$ are defined as

$$
\begin{aligned}
& J_{s 1}=\left[\begin{array}{ll}
\mathbf{I}_{N-1} & 0
\end{array}\right] \in R^{(N-1) \times N} \\
& J_{s 2}=\left[\begin{array}{ll}
\mathbf{0} & \mathbf{I}_{N-1}
\end{array}\right] \in R^{(N-1) \times N},
\end{aligned}
$$

where I is identity matrix. We define $Q_{N}$ as [10]

$$
\mathbf{Q}_{2 M}=\frac{1}{\sqrt{2}}\left[\begin{array}{cc}
\mathbf{I}_{M} & j \mathbf{I}_{M} \\
\boldsymbol{\Pi}_{M} & -j \boldsymbol{\Pi}_{M}
\end{array}\right]
$$

if $N=2 M$ or

$$
\mathbf{Q}_{2 M+1}=\frac{1}{\sqrt{2}}\left[\begin{array}{ccc}
\mathbf{I}_{M} & \mathbf{0} & j \mathbf{I}_{M} \\
\mathbf{0}^{T} & \sqrt{2} & \mathbf{0}^{T} \\
\boldsymbol{\Pi}_{M} & \mathbf{0} & -j \boldsymbol{\Pi}_{M}
\end{array}\right]
$$

if $N=2 M+1 . \Pi_{M}$ is the $M \times M$ exchange matrix with ones on its antidiagonal and zeros elsewhere. $\mathbf{0}$ is a column vector of zeros. $\mathbf{Q}_{N}^{H}$ is a sparse unitary matrix that transforms $v_{N}(\psi)$ into a real-valued manifold; $d_{N}(\psi)=\mathbf{Q}_{N}^{H} v_{N}(\psi)$, where the operator $(\cdot)^{H}$ denotes complex conjugate transpose. Then, it is easy to obtain

$$
\tan \left(\frac{\psi}{2}\right) \mathbf{K}_{1} d_{N}(\psi)=\mathbf{K}_{2} d_{N}(\psi) .
$$

$\mathbf{K}_{1}$ and $\mathbf{K}_{2}$ are defined as [11].

$$
\begin{aligned}
& \mathbf{K}_{1}=\operatorname{Re}\left\{\mathbf{Q}_{N-1}^{H} J_{s 2}^{(N)} \mathbf{Q}_{N}\right\} \\
& \mathbf{K}_{2}=\operatorname{Im}\left\{\mathbf{Q}_{N-1}^{H} J_{s 2}^{(N)} \mathbf{Q}_{N}\right\} .
\end{aligned}
$$

(2) Consider an $N \times \widehat{K}$ real-valued matrix

$$
\mathbf{V}_{R}(\psi)=\left[d_{N}\left(\psi_{k}\right)\right]_{k=1}^{\widehat{K}},
$$

where $\widehat{K}$ is the estimated number of scattering centers, which can be detected by Gerschgorin disk estimator (GDE) [12]. Then, substituting (7) into (5) implies the following relations:

$$
\mathbf{K}_{1} \mathbf{V}_{R}(\psi) \boldsymbol{\Omega}_{\psi}=\mathbf{K}_{2} \mathbf{V}_{R}(\psi),
$$

where

$$
\boldsymbol{\Omega}_{\psi}=\operatorname{diag}\left\{\tan \left(\frac{\psi_{k}}{2}\right)\right\}_{k=1}^{\widehat{K}} .
$$

(3) Let $\widehat{\mathbf{U}}_{\mathrm{RS}}=\mathrm{V}_{R} \mathrm{~T}$, where $\widehat{\mathrm{U}}_{\mathrm{RS}}$ represents the signal eigenvectors and $\mathrm{T}$ is an unknown $\widehat{K} \times \widehat{K}$ real-valued matrix. Substituting $\mathbf{V}_{R}=\widehat{\mathbf{U}}_{\mathrm{RS}} \mathbf{T}^{-1}$ into (8) yields the signal eigenvector relations

$$
\mathbf{K}_{1} \widehat{\mathbf{U}}_{\mathrm{RS}} \Psi=\mathbf{K}_{2} \widehat{\mathbf{U}}_{\mathrm{RS}}
$$

with

$$
\widehat{\boldsymbol{\Psi}}=\mathbf{T}^{-1} \boldsymbol{\Omega}_{\psi} \mathbf{T}
$$

and $\widehat{\Psi}$ can be solved with least square criterion.

(4) Compute $\left.\hat{\lambda}_{k}\right|_{k=1} ^{\widehat{K}}$ as the eigenvalues of $\widehat{\boldsymbol{\Psi}}$, and the spatial frequencies are estimated as

$$
\widehat{\psi}_{k}=2 \tan ^{-1}\left\{\widehat{\lambda}_{k}\right\}_{k=1}^{\widehat{K}} .
$$

According to (12) the estimation of $\widehat{r}_{k}$ is

$$
\widehat{r}_{k}=\frac{\widehat{\psi}_{k}}{(-4 \pi \Delta f / c)},
$$

where $r_{k}$ is the radial location of the scattering centers and $\Delta f$ is frequency step.

(5) The last step is amplitude estimation, which can be accomplished through least square criterion by means of the estimated locations of the target.

2.2. HRRP Reconstruction. Assume that there are $K$ point scattering centers along the down range locating at different $r_{k}$ locations, and the backscattered field at the far field can be approximated as [13].

$$
X(f)=\sum_{k=1}^{K} \sigma_{k} \exp \left(\frac{-j 4 \pi r_{k} f}{c}\right),
$$

where $f$ is the frequency, $c$ is the velocity of light, and $\sigma_{k}$ and $r_{k}$ are the scattering strength and down range of the $k$ th scattering center, respectively. Once the scattering centers are extracted, the reverse process, that is, reconstruction of the HRRP, can be readily done in real time by means of the extracted scattering centers.

$$
\widehat{X}(f)=\sum_{k=1}^{\widehat{K}} \widehat{\sigma}_{k} \exp \left(\frac{-j 4 \pi \widehat{r}_{k} f}{c}\right),
$$

where $\widehat{\sigma}_{k}$ and $\widehat{r}_{k}$ are the estimated scattering strength and down range of the $k$ th scattering center, respectively. To summarize, it has been shown that a sparse model based on 


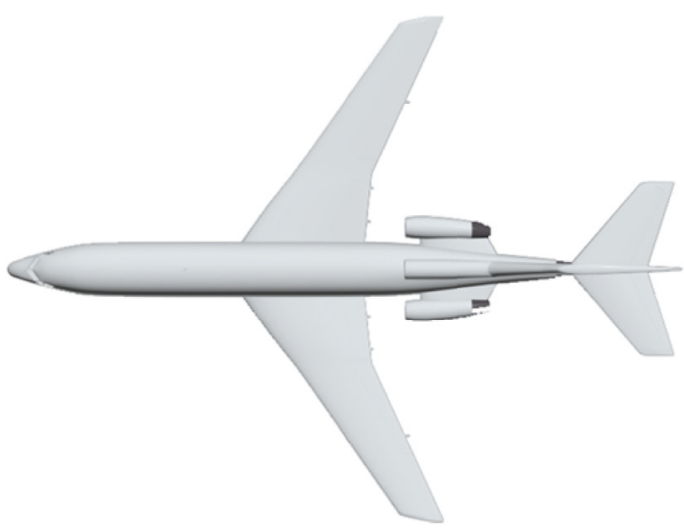

(a)

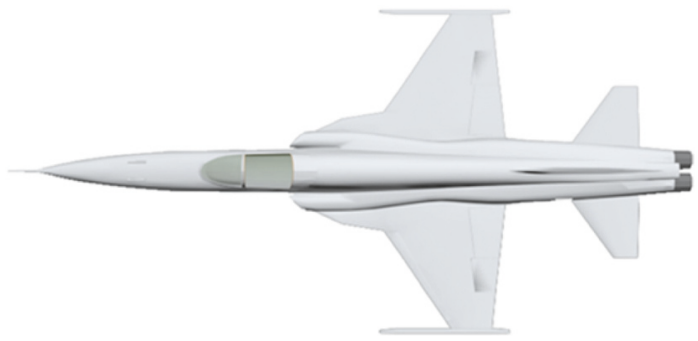

(c)

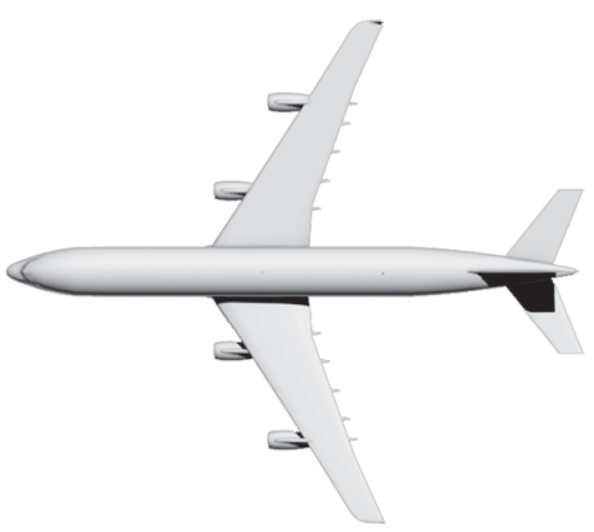

(b)

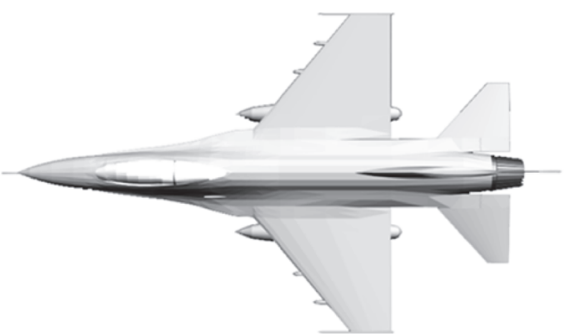

(d)

Figure 1: Four 3D models of the targets: (a) Target 1, (b) Target 2, (c) Target 3, and (d) Target 4.

scattering center representation can be constructed. Furthermore, this model can be used to reconstruct HRRP with a very fine resolution and good fidelity.

2.3. Feature Extraction Based on Central Moments. Feature analysis and extraction play significant role in HRRP ATR. Central moments are available for this issue, and they were firstly used to describe the features of two-dimensional (2D) image signal [14]. They are invariant against translation, rotation, and scaling and are suitable for the representation of any shape [15]. In this study, we only use translational and scale invariance to recognize one-dimensional scattering center patterns, which is available using central moments [16]. Next, central moment features of normalized range profiles are computed to provide translational invariance. After the normalization of amplitude of each scattering center with the maximum amplitude, the $p$ th order central moment of the given one-dimensional scattering center distribution can be computed by [17]

$$
\mu_{p}=\sum_{k=1}^{L}\left(\bar{r}_{k}-\bar{\eta}_{r}\right)^{p}\left[\frac{\bar{a}_{k}}{\sum_{k=1}^{L} \bar{a}_{k}}\right], \quad \bar{r}_{k} \in[0,1]
$$

where

$$
\begin{aligned}
& \bar{\eta}_{r}=\sum_{k=1}^{L} \bar{r}_{k}\left[\frac{\bar{a}_{k}}{\sum_{k=1}^{L} \bar{a}_{k}}\right] \\
& \bar{r}_{k}=\frac{r_{k}}{R_{u}}, \quad k=1,2, \ldots, L
\end{aligned}
$$

$$
\bar{a}_{k}=\frac{\left|a_{k}\right|}{\left|a_{k}\right|_{\max }}, \quad k=1,2, \ldots, L
$$

and $\left|a_{k}\right|_{\max }$ is the maximum magnitude in the estimated $L$ amplitudes $\left|a_{k}\right|$ from range profile vectors. $R_{u}$ is the maximum unambiguous range. Using the central moments in (16), the feature vector $F$ can be represented as

$$
F=\left[\mu_{1}, \mu_{2}, \ldots, \mu_{p_{\max }}\right]^{T},
$$

where $p_{\max }$ is the maximum order of central moments used to form a feature vector, which is set to 20 in this paper.

2.4. SARPROP Neutral Network. Classification algorithms based on feed-forward neural networks play an important role in radar HRRP target recognition. Therefore, in the classification phase, we use the SARPROP Neutral Network classification algorithm to evaluate the recognition performances of FFT based HRRP and reconstructed HRRP. Although gradient descent is the most commonly used method for training feed-forward neural networks, it suffers from the problem of converging to local minima with nonideal performance. This problem can be overcome through the use of global optimization [18]. By combining gradient descent with the global optimization technique of simulated annealing (SA), SARPROP [19] can not only escape local minima but also maintain and improve the training times of the resilient backpropagation (RPROP) algorithm. Reference [20] is available for more details about SARPROP algorithm. In this paper, we used similar parameters setting as [20]. 


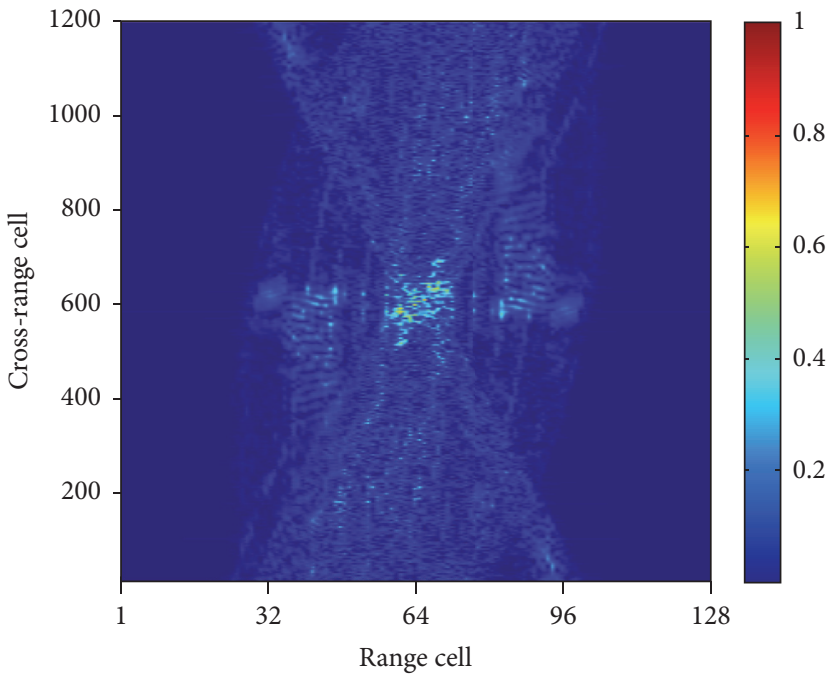

(a)

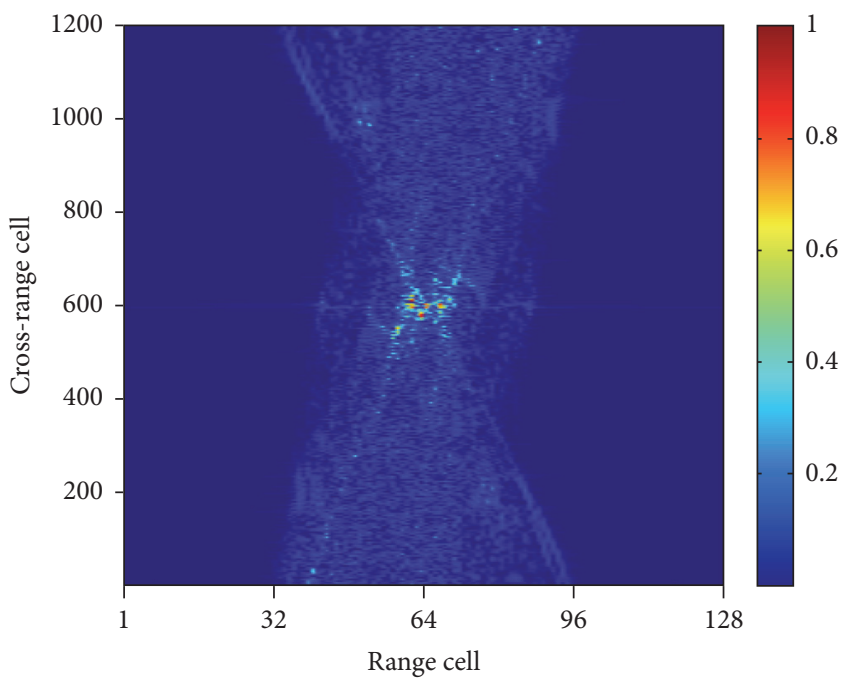

(c)

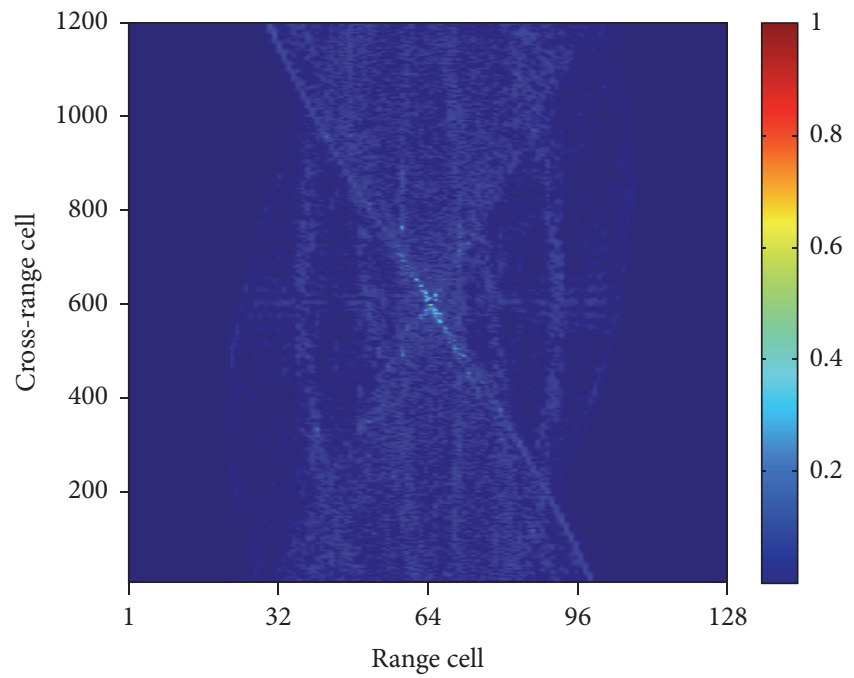

(b)

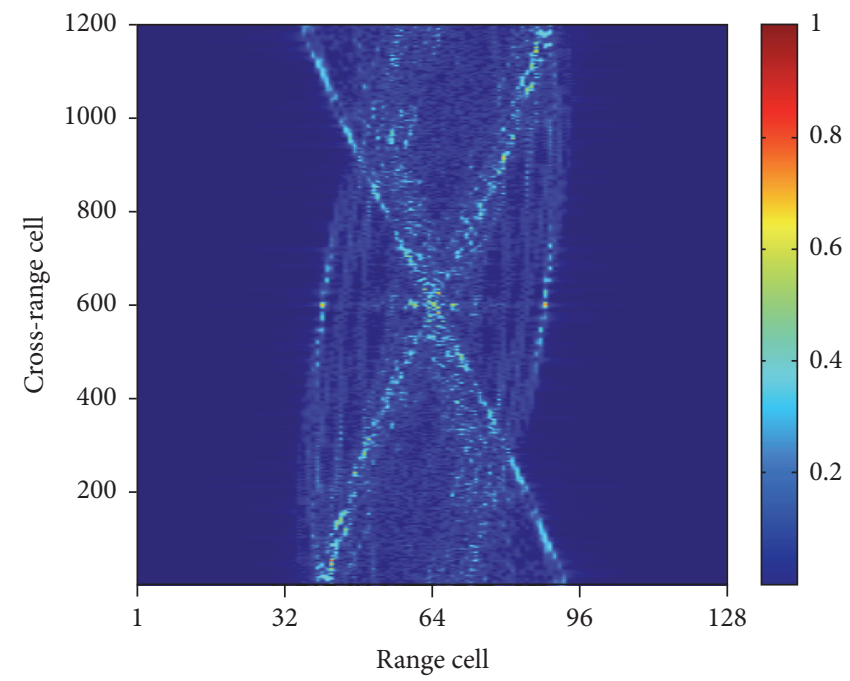

(d)

Figure 2: Normalized range profiles obtained by FFT with no noise: (a) Target 1, (b) Target 2, (c) Target 3, and (d) Target 4.

\section{Simulation Results}

3.1. Data Description. The $2 \mathrm{D}$ backscatters distribution data of four different scaled aircraft models (e.g., Targets 1, 2, 3, and 4) are used in the simulation. The range profiles are from aspects that vary from 0 to 180 degrees ( 0 is head direction) with an interval of 0.5 degrees. Therefore, the data set consists of 1440 samples altogether for four targets. The three-dimensional (3D) models of the targets are illustrated in Figure 1.

The radar transmitter sends out stepped frequency at starting frequency of $3.25 \mathrm{GHz}$ and $\mathrm{HH}$ polarization was used. Note that the size of each target is merely about 50 wavelengths. The frequency bandwidth is $400 \mathrm{MHz}$ and pulse duration of single pulse is $25.6 \mu \mathrm{s}$. A total of 128 stepped frequency waveforms are transmitted at each azimuth. Before target recognition experiments, the data set for each aircraft model is divided into training subset and test subset in the proportion of $1: 6$. The training data are selected by the equal interval partition method with each azimuth containing 50 HRRP samples, resulting in the total of 200 samples. The test set at each signal-to-noise ratio (SNR) consists of 300 range profiles for each target, thus with 1200 samples in total. Hence the training set size is only $16.67 \%$ of the overall data.

3.2. HRRP Reconstruction Results. SNR is a key factor that impacts the recognition performance. In this section, Gaussian noise is added to the simulated target signature data with certain SNR defined by

$$
\mathrm{SNR}=10 \times \log 10\left(\frac{E_{s}}{\sigma_{n}^{2}}\right),
$$

where $E_{s}$ is the total energy of the frequency domain data and $\sigma_{n}^{2}$ is the variance of the additive noise.

It can be observed from Figure 2 that the normalized range profiles using FFT is simulated, and the HRRP at 


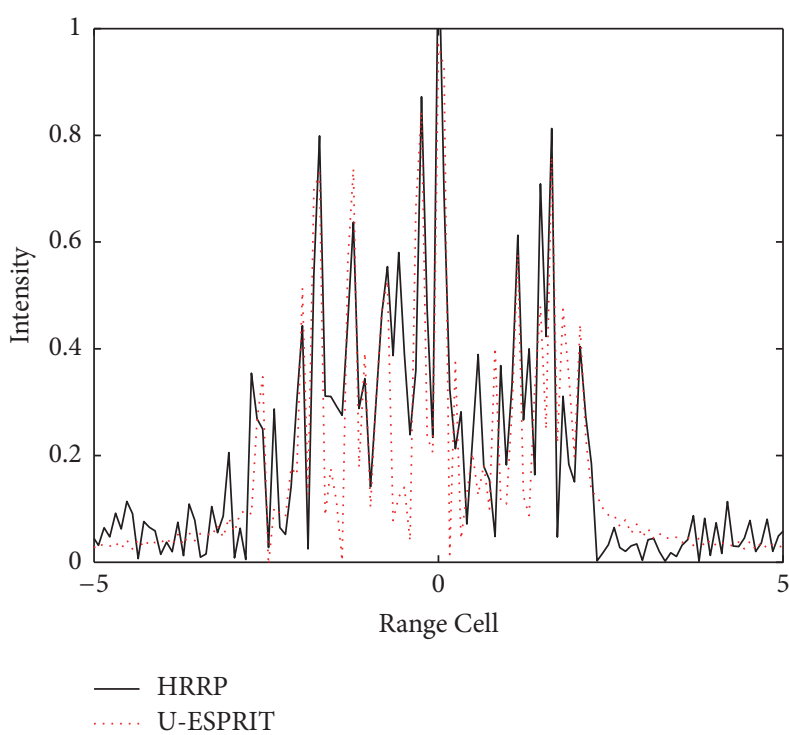

(a)

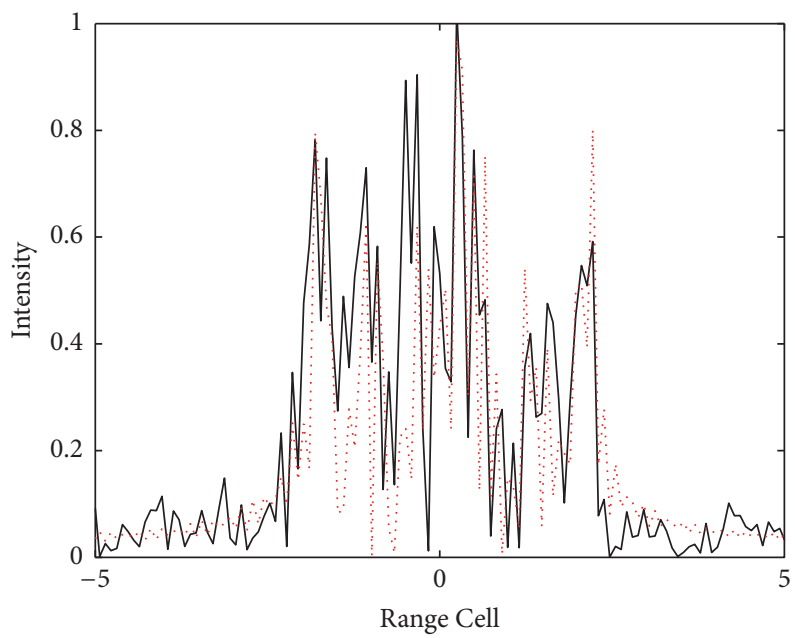

$\begin{array}{ll}\text { —... HRRP } & \text { U-ESPRIT }\end{array}$

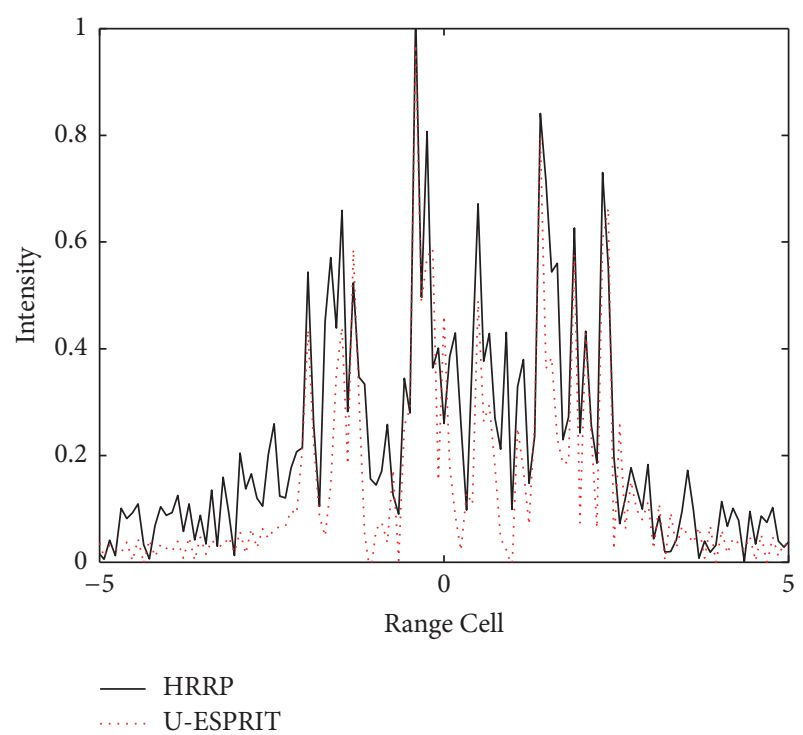

(b)

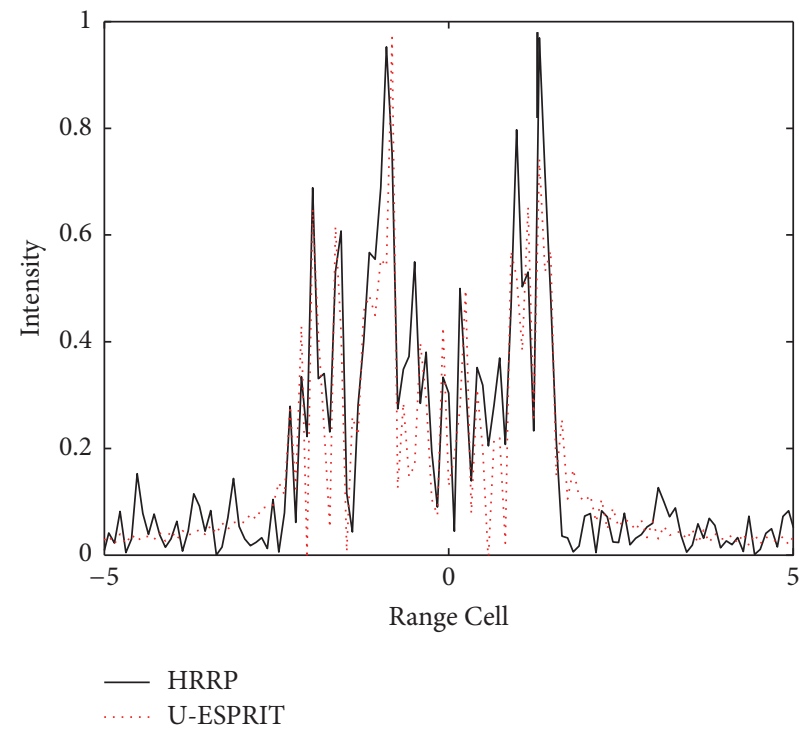

(d)

FIGURE 3: Normalized range profiles obtained by unitary ESPRIT and FFT with SNR equaling $5 \mathrm{~dB}$ at an azimuth of 30 degrees: (a) Target 1, (b) Target 2, (c) Target 3, and (d) Target 4.

each azimuth is reconstructed using the unitary ESPRIT based method. An example (at azimuth of 30 degrees) is given in Figure 3. From the reconstructed results, we can conclude that the proposed method could not only rebuild the superresolution HRRP but also reduce the adaptive noise efficiently.

3.3. Recognition Performance Comparison against Noise. After each HRRP is reconstructed successfully, the recognition procedure can be readily done. To assess the feasibility and accuracy of the proposed algorithm in enhancing the classification rate of HRRP, we apply SARPROP to reconstructed HRRP classification and evaluate its performance compared with directly using HRRP. The testing samples of the four aircraft models are contaminated by independent additive white Gaussian noise (AWGN) to achieve the desired SNR from 0 to $50 \mathrm{~dB}$ with a $10 \mathrm{~dB}$ interval. To guarantee the accuracy of the recognition rate, we performed 20 times Monte Carlo simulations. It is demonstrated that reconstructed HRRP has better antinoise performance and greater classification potential than original HRRP. As shown in Figure 4, the reconstructed HRRP can achieve higher classification rates than original HRRP in all different SNR scenarios. It also shows that the performance of the proposed method is independent of SNR.

\section{Conclusion}

We presented a new noise-robust method for the radar HRRP recognition by means of unitary ESPRIT to improve the 

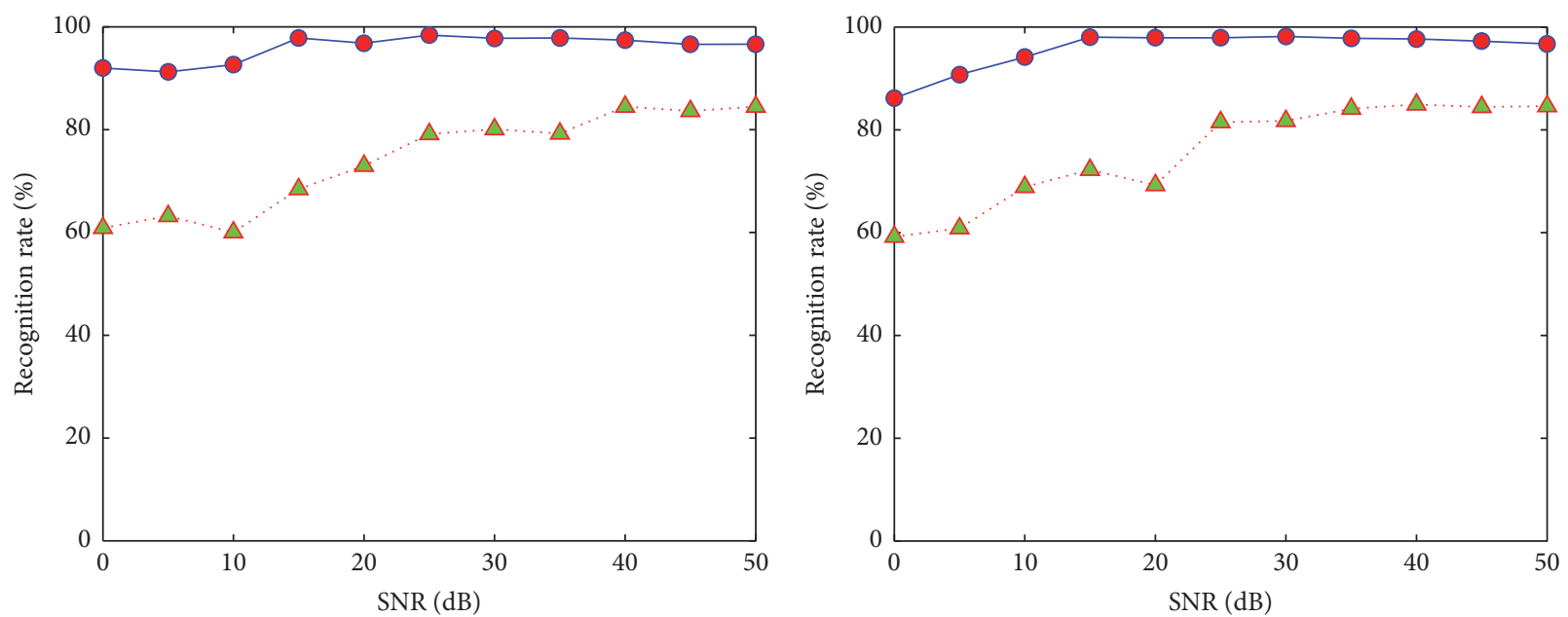

. $\triangle$. HRRP

- Unitary ESPRIT

. $\triangle$. HRRP

- Unitary ESPRIT

(a)
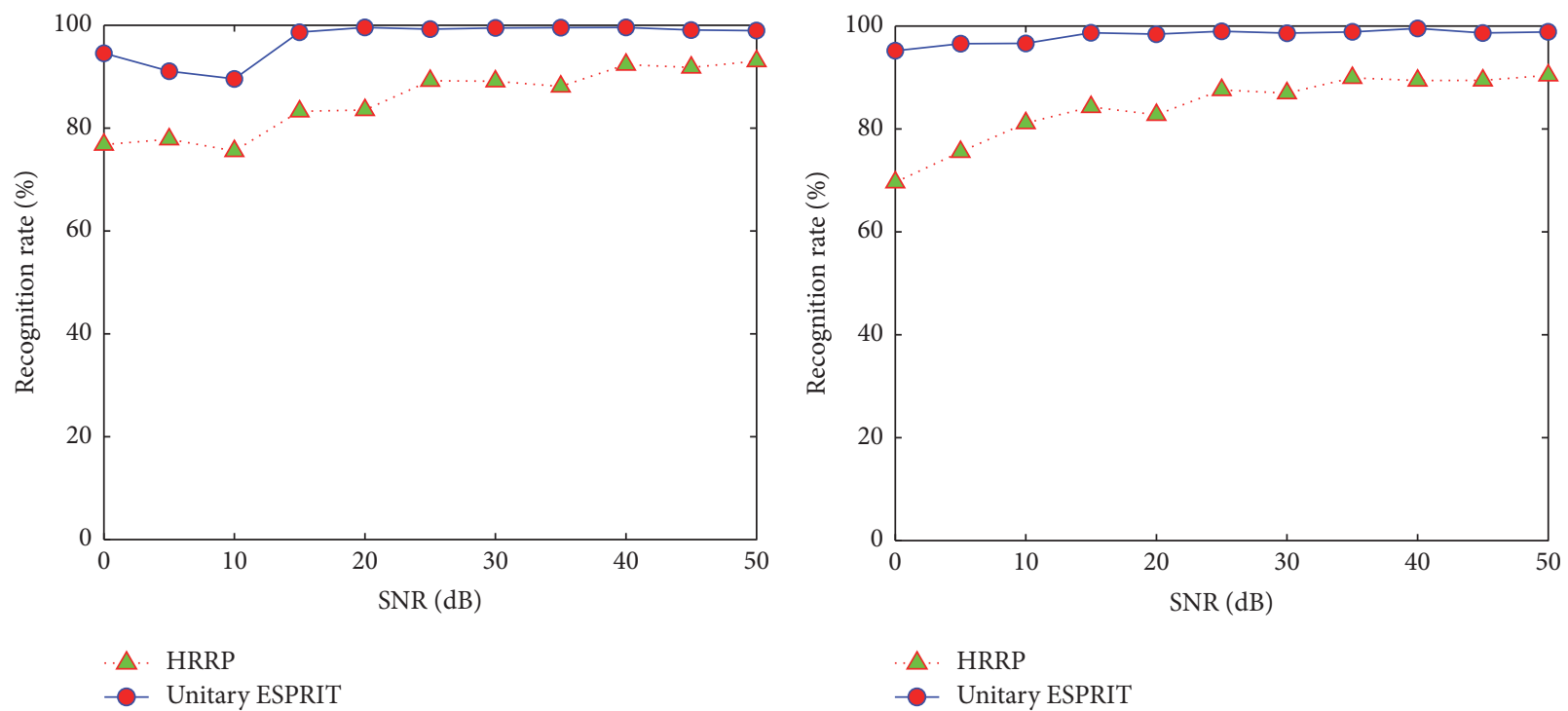

(c)

(d)

FIGURE 4: The average recognition rates of original method and our method for four targets versus SNR: (a) Target 1, (b) Target 2, (c) Target 3 , and (d) Target 4.

classification performance. The proposed method focused on the improvement of the classification performance through enhancing the resolution and reducing storage burden. The method would use unitary ESPRIT method to extract the scattering centers accurately along the aspect in the first stage and reconstruct the HRRP by means of the extracted scattering centers, that is, locations and amplitudes in the second stage. Experimental results showed that the proposed classification scheme could achieve better antinoise performance than the original method and has potential in radar HRRP classification. By adopting SARPROP Neutral Network, targets can be effectively classified via employing the reconstructed HRRP, thereby improving systems accuracy.

\section{Conflicts of Interest}

The authors declare that there are no conflicts of interest regarding the publication of this paper.

\section{Acknowledgments}

This work was supported by the Fundamental Research Funds for the Central Universities and the National Natural Science Foundation of China under Grant no. 61372004. 


\section{References}

[1] H.-J. Li and S.-H. Yang, "Using range profiles as feature vectors to identify aerospace objects," IEEE Transactions on Antennas and Propagation, vol. 41, no. 3, pp. 261-268, 1993.

[2] K.-T. Kim, D.-K. Seo, and H.-T. Kim, "Efficient radar target recognition using the MUSIC algorithm and invariant features," IEEE Transactions on Antennas and Propagation, vol. 50, no. 3, pp. 325-337, 2002.

[3] R. O. Schmidt, "Multiple emitter location and signal parameter estimation," IEEE Transactions on Antennas and Propagation, vol. 34, no. 3, pp. 276-280, 1986.

[4] J. Capon, "High-resolution frequency-wavenumber spectrum analysis," Proceedings of the IEEE, vol. 57, no. 8, pp. 1408-1418, 1969.

[5] E. K. Walton, "Far-field measurements and maximum entropy analysis of lossy material on a conducting plate," IEEE Transactions on Antennas and Propagation, vol. 37, no. 8, pp. 1042-1047, 1989.

[6] R. Carrière and R. L. Moses, "High resolution radar target modeling using a modified prony estimator," IEEE Transactions on Antennas and Propagation, vol. 40, no. 1, pp. 13-18, 1992.

[7] Y. D. Guo, Y. S. Zhang, and N. N. Tong, "Beamspace ESPRIT algorithm for bistatic MIMO radar," Electronics Letters, vol. 47, no. 15, pp. 876-878, 2011.

[8] M. Haardt, M. D. Zoltowski, C. P. Mathews, and J. A. Nossek, "2D unitary ESPRIT for efficient 2D parameter estimation," in Proceedings of the 1995 20th International Conference on Acoustics, Speech, and Signal Processing, vol. 3, pp. 2096-2099, May 1995.

[9] M. D. Zoltowski, M. Haardt, and C. P. Mathews, "Closed-form 2-D angle estimation with rectangular arrays in element space or beamspace via unitary ESPRIT,' IEEE Transactions on Signal Processing, vol. 44, no. 2, pp. 316-328, 1996.

[10] K.-C. Huarng and C.-C. Yeh, "A unitary transformation method for angle-of-arrival estimation," IEEE Transactions on Signal Processing, vol. 39, no. 4, pp. 975-977, 1991.

[11] H. L. V. Trees, "Optimum array processing part IV," in Detection, Estimation, and Modulation Theory, pp. 946-948, Jone Wiley \& Sons, New York, NY, USA, 2002.

[12] H.-T. Wu, J.-F. Yang, and F.-K. Chen, "Source number estimators using transformed Gerschgorin radii," IEEE Transactions on Signal Processing, vol. 43, no. 6, pp. 1325-1333, 1995.

[13] Özdemir C., Inverse Synthetic Aperture Radar Imaging With MATLAB Algorithms, Jone Wiley \& Sons, Hoboken, NJ, USA, 2011.

[14] J. Flusser, T. Suk, and S. Saic, "Recognition of blurred images by the method of moments," IEEE Transactions on Image Processing, vol. 5, no. 3, pp. 533-538, 1996.

[15] S. Luo and S. Li, "Automatic target recognition of radar HRRP based on high order central moments features," Journal of Electronics, vol. 26, no. 2, pp. 184-190, 2009.

[16] M. K. Hu, "Visual pattern recognition by moment invariant," IRE Transactions of Information Theory, vol. 8, no. 2, pp. 179187, 1962.

[17] X. Liu, M. Gao, and X. Fu, "Application of HRRP even rank central moments features in satellite target recognition," in Proceedings of the RADAR 2007 - The Institution of Engineering and Technology International Conference on Radar Systems, pp. 1-4, October 2007.
[18] N. K. Treadgold and T. D. Gedeon, "The SARPROP algorithm: a simulated annealing enhancement to resilient back propagation," in Proceedings the Int . Panel Conf. Soft and Intell. Comput, pp. 289-293, Budapest, Hungary, 1996.

[19] N. K. Treadgold and T. D. Gedeon, "Simulated annealing and weight decay in adaptive learning: The SARPROP algorithm," IEEE Transactions on Neural Networks, vol. 9, no. 4, pp. 662668, 1998.

[20] M. Riedmiller and H. Braun, "A direct adaptive method for faster backpropagation learning: the RPROP algorithm," in Proceedings of the IEEE International Conference on Neural Networks (IJCNN '93), vol. 1, pp. 586-591, IEEE, San Francisco, Calif, USA, March-April 1993. 


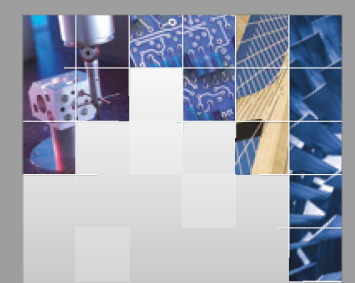

\section{Enfincering}
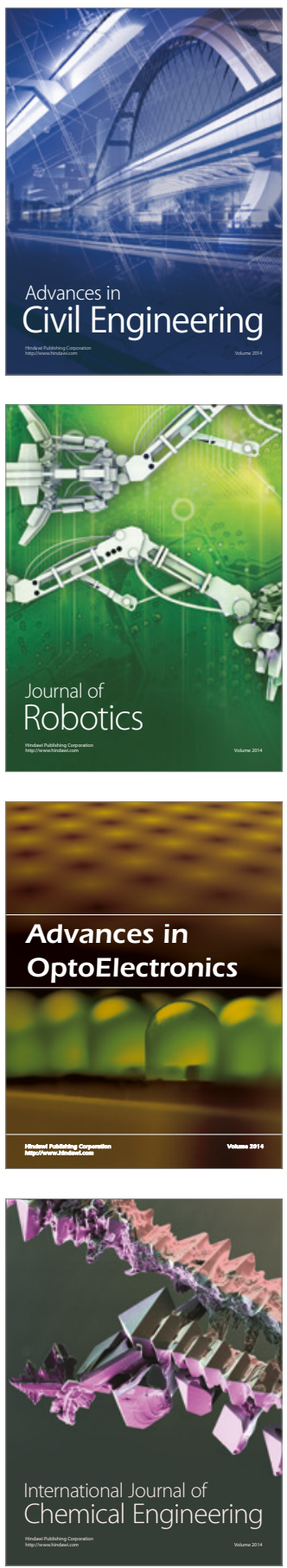

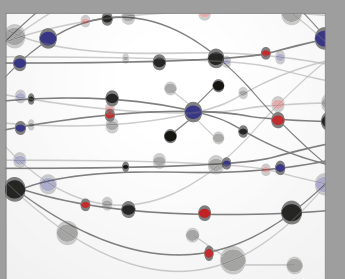

The Scientific World Journal

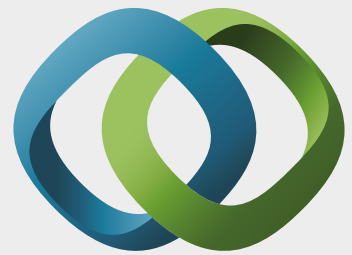

\section{Hindawi}

Submit your manuscripts at

https://www.hindawi.com
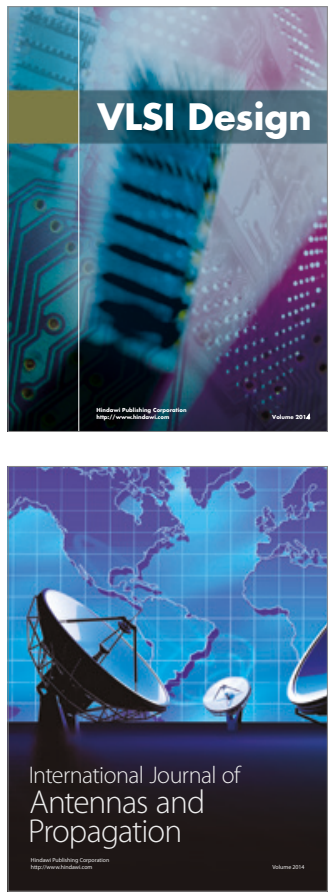

\section{Rotating}

Machinery
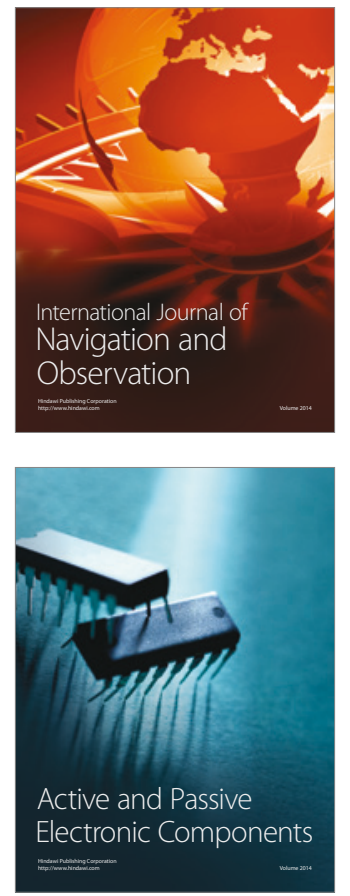
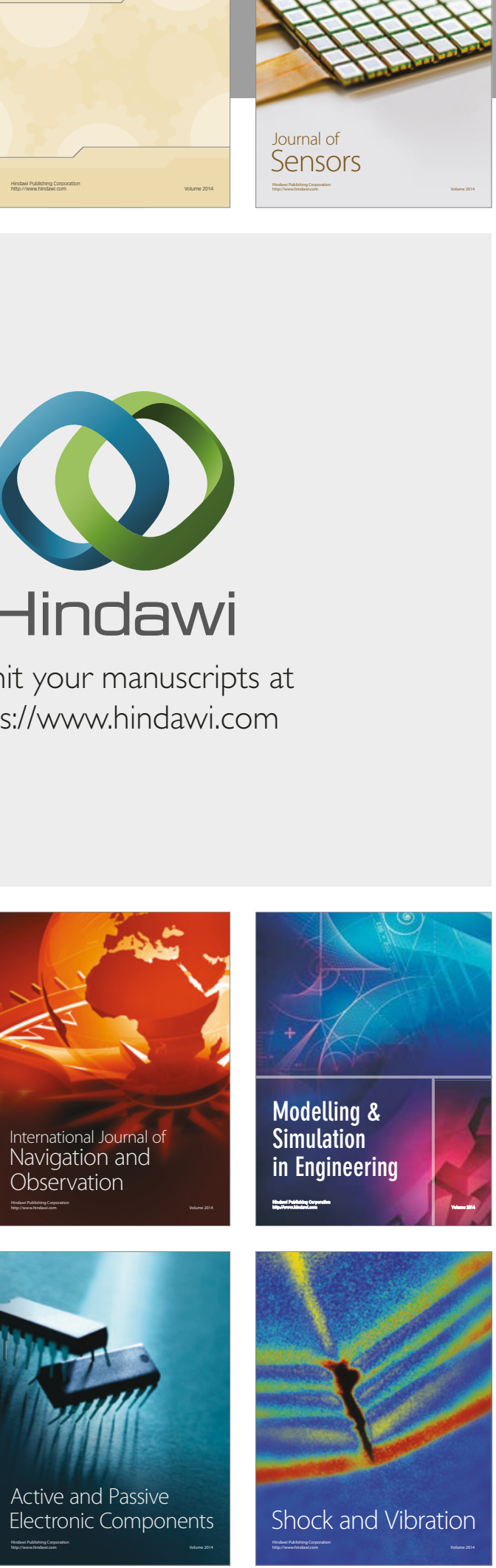
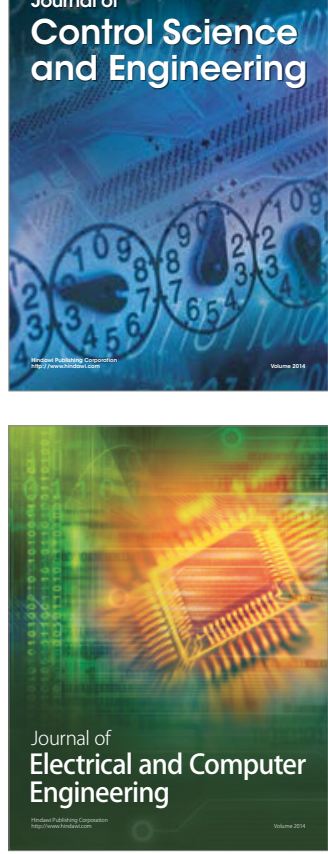

Distributed

Journal of

Control Science

and Engineering
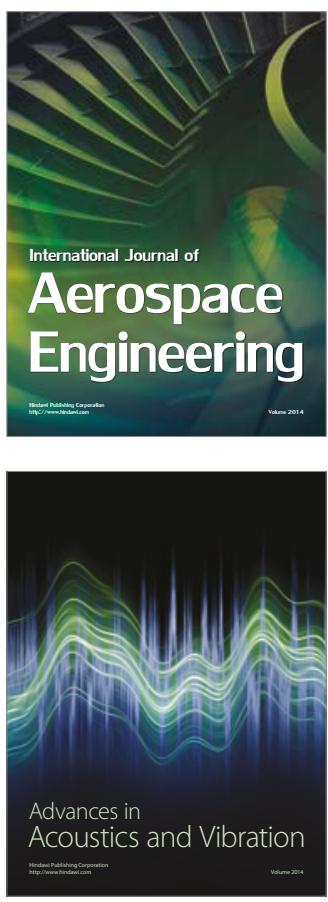

Sensor Networks 